\title{
Cylindrically-Collimated, Self-Similar MHD Disk Outflows
}

\author{
Eve C. Ostriker ${ }^{1}$ \\ Harvard-Smithsonian Center for Astrophysics, Cambridge, MA, USA
}

\begin{abstract}
We describe the dynamics of a class of MHD winds from Keplerian-rotating disks. In this model, all flow velocities are assumed to vary self-similarly with spherical radius $r$ as $v(r, \theta) \propto r^{-1 / 2}$, with density varying as $\rho(r, \theta) \propto r^{-q}$ for arbitrary $q$. At large distances from the disk, the wind is explicitly required to become cylindrically collimated. We find that the asymptotic wind solution has power-law scalings of all flow variables in the cylindrical radius $R=r \sin \theta$, and $q<1$ is necessary. We describe how the Alfvén criticality condition limits the space of energy and angular momentum parameters defining these wind solutions. We present an example of the run of density, velocity, and magnetic field for a full solution of the wind equations, and compare the properties of these cylindrically-collimated wind solutions to previous work.
\end{abstract}

\section{Introduction}

Observations of jets and outflows from young stellar systems evidence strong collimation in both density and velocity, with some jet systems appearing close to cylindrically-collimated far from the source (e.g. Ray et al 1996, Lada \& Fich 1996, Bachiller \& Gomez-Gonzalez 1992) A well-established theoretical way of producing a fast wind is magnetocentrifugal driving from a rapidly-rotating star or accretion disk (see e.g. review of Königl \& Ruden 1993). Heyvaerts \& Norman (1989) showed that under certain conditions, at large distances from the source of an MHD outflow, the projections of streamlines must assume either cylindrical or "parabolic" loci (in the former case a streamline's cylindrical radius $R \rightarrow$ constant as the height above the disk $z \rightarrow \infty$; in the latter case $R \rightarrow \infty$ and $R / z \rightarrow 0$ as $z \rightarrow \infty$ ).

When an accretion disk has a large dynamic range of radii between inner and outer boundaries, and considerable ordered magnetic flux can be advected into the disk, the outflow may assume a self-similar configuration away from the boundaries. Blandford and Payne (1982; hereafter BP) developed the first $r$ self-similar MHD wind solutions, starting from the ansatz $v \propto r^{-1 / 2}, \rho \propto r^{-3 / 2}$, and $B \propto r^{-5 / 4}$ for the dependence, respectively, of all velocities, the mass density, and the magnetic field strength with spherical radius $r$ from the center. More recently, a number of authors (e.g. Begelman, Chiueh, Contopoulos, Fer-

${ }^{1}$ present address: Department of Astronomy, The University of Maryland, College Park, MD 20742-2421, USA 
reira, Königl, Li, Lovelace, Pelletier, Pudritz, Rosso, Sauty, Trussoni, Tsinganos, Wardle) have extended and generalized the BP models. Developing idealized solutions of this sort has provided a practical way to learn about many generic properties of MHD winds.

A difficulty of the original BP solutions is their behavior near the polar axis. While BP halted their integrations short of the pole, they argued that for most parameter choices, the streamlines in their solutions would reach a maximum cylindrical radius before turning around and recollimating toward the axis (for special sets of parameters, parabolic solutions which become fast-magnetosonic at infinite distance are also expected). Integrations by other workers have subsequently verified that the BP-type solutions do recollimate, and have also produced oscillatory solutions for other parameter regimes (e. g. Contopoulos \& Lovelace 1994). Previous studies, however, have not attempted to match to specific boundary conditions at the pole; instead, integrations which are initiated at the equator are halted at either an arbitrary point, or when the solution becomes singular near the "modified fast magnetosonic" point where $v_{6} / v_{A}=1$ (e. g. Ferreira 1996).

The goal of the present work is to construct $r$-self-similar outflow solutions which have a prescribed non-singular asymptotic behavior at the pole, such that they could in principle be embedded in a larger global solution with uniform media inside and outside the wind. At the pole, we demand that the poloidal projection of the solution streamlines be vertical, corresponding to cylindricallycollimated outflows. For more details of our results see Ostriker (1996).

\section{Analysis}

To generalize the BP models, we look for solutions of the ideal, cold MHD equations with $v \propto r^{-1 / 2}, \rho \propto r^{-q}$, and $B \propto r^{-(1+q) / 2}$. With this assumption, the mass flow, momentum flow, and energy flow in the wind scale with $r$ as $\dot{M} \propto$ $r^{3 / 2-q}, \dot{P} \propto r^{1-q}$, and $\dot{E} \propto r^{1 / 2-q}$. When $q<3 / 2$ the mass flux is dominated by the outer disk; when $q<1(q>1)$ the momentum flux is dominated by the outer (inner) disk; when $q>1 / 2$, the energy flux is dominated by the inner disk. To match the desired cylindrical boundary conditions, we need an analytic asymptotic solution. If we use the ansatz $v \propto R^{-1 / 2}, \rho \propto R^{-q}$, and $B \propto$ $R^{-(1+q) / 2}$ with $R=r \sin \theta$, we indeed find a self-consistent lowest-order solution to the MHD equations at the pole. The next two orders in the approximation for the flow variables are obtained using Frobenius series expansions; we then begin a numerical integration.

In our solutions, each magnetic field line rotates at the Kepler rotation rate $\Omega$ corresponding to its footpoint $R_{0}$ in the disk. The flow on a streamline makes an Alfvén transition $\left(v_{A, p}=v_{p}\right)$ at cylindrical radius $R_{A}$, and lateral expansion asymptotes to cylindrical radius $R_{1}$. For fixed $q$, the asymptotic selfsimilar cylindrical solutions are characterized by two parameters, $j \equiv J / \Omega R^{2}=$ $\left(R_{A} / R_{1}\right)^{2}$ and $e \equiv-\mathcal{E} /(\Omega R)^{2}=(3 / 2)\left(R_{0} / R_{1}\right)^{2}$. Here, the total specific angular momentum of the flow (including material and magnetic terms) is

$$
J=R\left(v_{\varphi}-\frac{B_{\varphi} B_{p}}{4 \pi \rho v_{p}}\right)
$$



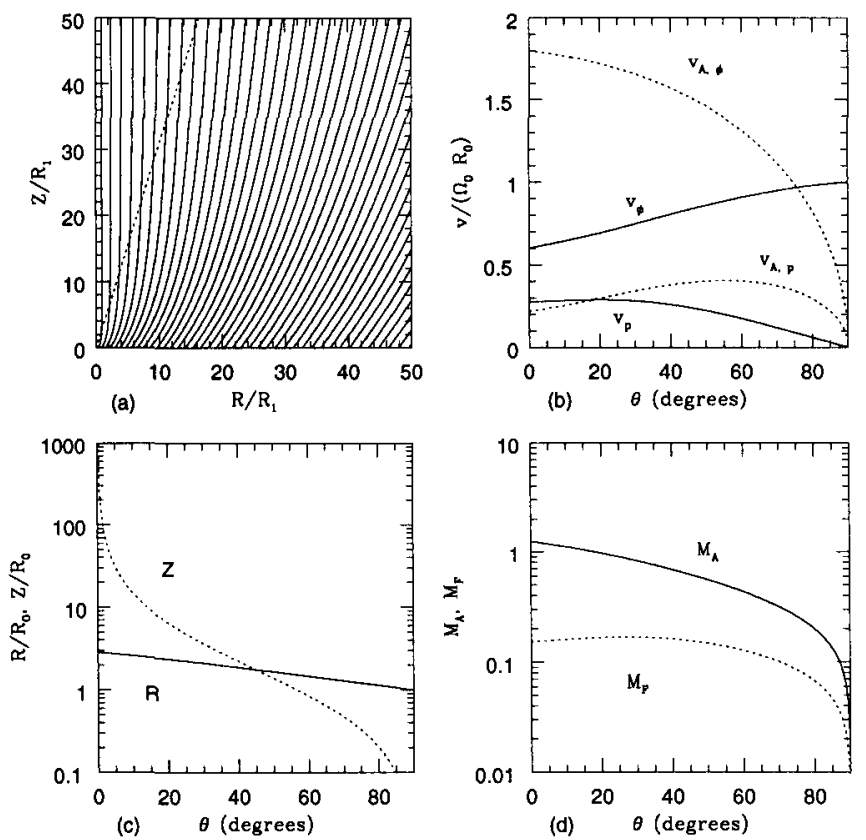

Figure 1. Flow solution for $\mathrm{q}=0.75, R_{0} / R_{1}=0.35, R_{A} / R_{0}=2.41$. (a) Streamlines (solid curves) for equal increments in $\dot{M}$. The innermost streamline originates at $R / R_{1} \equiv R_{0} / R_{1}=0.35$ and asymptotes to $R / R_{1}=1$. The dashed line shows the locus of the Alfvén surface. (b) Fluid speeds (solid curves) and Alfvén speeds (dashed curves) of poloidal and toroidal flow/field components, in units of the Kepler speed $\Omega_{0} R_{0}$ at the streamline footpoint, as a function of angle $\theta$ with respect to the pole. (c) Streamline radial distance (solid curve) and height above the disk (dashed curve) in units of the footpoint radius $R_{0}$. (d) Alfvén-mode (solid curve) and fast-mode (dashed curve) Mach numbers $M_{A} \equiv v_{p} / v_{A, p}$ and $M_{F} \equiv v_{p} / v_{A}$ for the flow.

and the Bernoulli parameter is

$$
\mathcal{E}=\frac{1}{2}|\mathbf{v}|^{2}-\Omega R v_{\varphi}-\frac{G M_{*}}{r}
$$

with the gravity of a central point mass $M_{*}$ assumed. It turns out that the self-similar cylindrical solutions exist only when the scaling parameter $q<1$ so that the magnetic tension is large enough to offset the centrifugal force and magnetic pressure gradient; i.e. both density and magnetic field strength must decrease outward more slowly than $r^{-1}$. Further constraints on the cylindrical solutions are described in Ostriker (1996). 


\section{Wind solutions and discussion}

While the cylindrical solutions which represent the polar boundary condition for our winds are described by a three-parameter family, only a subset of these cylindrical flows can be realized by a smooth acceleration away from a disk. The added constraint of a smooth Alfvén transition reduces the solution space to a two parameter family. Numerically, we obtain solutions by specifying $q$ and $R_{0} / R_{1}$ and then searching for $R_{A} / R_{0}$ such that an integration from the pole passes smoothly through the Alfvén point and ends on the disk.

Figure 1 shows streamline geometry and the variation of flow quantities along streamlines for an example of a wind solution. Like the rest of the solutions we have obtained, this solution shows no oscillations in the crossectional radius, instead having a secular increase in $R$ with $z$. The fast-mode Mach number $M_{F} \equiv v_{p} / v_{A}$ remains smaller than unity throughout the flow. Also, the toroidal component of the Alfven speed rises rapidly as the flow accelerates away from the disk, and in fact exceeds the poloidal component of the Alfven speed throughout. These features, which differ from those of the BP-type "overcollimating" solutions with $q>1$, hold for all of our cylindrically-collimated solutions (Ostriker 1996). The comparatively weak poloidal fields of these solutions do not enforce near-rigid rotation near the disk and centrifugally fling material to large radii; instead, the immediate toroidal field winding leads to a magnetic pressure gradient which accelerates the flow primarily vertically.

Our wind solutions have typical asymptotic flow speeds of some tenths of the Kepler speed $\Omega R_{0}$ at the streamline footpoint, rather than several times $\Omega R_{0}$ as in previous non-cylindrically-collimating solutions (e. g. Contopoulos \& Lovelace 1994). Since our solutions have $\dot{M}_{w} \propto R^{3 / 2-q}$ with $q<1$, they do however correspond to outflows where considerable mass is lost in the outer parts of the disk. Our typical asymptotic rotation speeds are a few to several tenths of $\Omega R_{0}$, and typical asymptotic Alfvén speeds are a few times $\Omega R_{0}$. For a fixed ratio between the Alfvén radius and the footpoint radius $R_{A} / R_{0}$, we find that as the matter and field distributions become less concentrated ( $q$ decreasing), the asymptotic poloidal speed increases, while the asymptotic cylindrical radius $R_{1}$ moves closer to the footpoint $R_{0}$.

\section{References}

Bachiller, R. \& Gomez-Gonzalez, J. 1992 Astronomy and Astrophysics Review, 3,257

Blandford, R. D. \& Payne, D. G. 1982, MNRAS, 199, 883

Contopoulos, J. \& Lovelace, R. V. E. 1994, ApJ429, 139

Ferreira, J. 1996, A\&A, in press

Heyvaerts, J. \& Norman, C. A. 1989, ApJ, 347, 1055

Königl A., \& Ruden, S. P. 1993, in Protostars and Planets III, eds. E. H. Levy and J. I. Lunine, Tucson: U. Arizona Press, 641

Lada, C. J. \& Fich, M. 1996, ApJ, 459, 638

Ray, T. P., Mundt, R., Dyson, J. E., Falle, S. A. E. G., \& Raga, A. C. 1996, ApJ, 468, L103 